\title{
Full-Band Signal Extraction from Sensors in Extreme Environments: The NASA InSight Microseismometer
}

\author{
Alexander E. Stott, Student Member, IEEE, Constantinos Charalambous, Tristram Warren, and William T. Pike
}

\begin{abstract}
Physically meaningful signal extraction from sensors deployed in extreme environments requires a combination of attenuation of confounding inputs and the removal of their residual using decorrelation techniques. In space applications where the resources for physical attenuation are limited, there is a necessity to apply the most effective post-processing analysis available. This paper describes the extraction of the seismic signal from a MEMS microseismometer to be deployed on the surface of Mars. The signal processing, which covers the full bandwidth $1 \times 10^{-5} \mathrm{~Hz}$ to $40 \mathrm{~Hz}$, uses a novel application of sensor fusion through an indirect Kalman Filter in combination with a thermal model of the microseismometer to remove the aseismic contribution of temperature over the frequency range. Owing to the full-band decorrelation, the analysis (based on pre-landing testing in analogous scenarios) produces both a characterisation of the microseismomter and a signal processing approach for information retrieval on Mars, along with other planetary and terrestrial planetary deployments.
\end{abstract}

Index Terms-Sensor fusion, MEMS seismometer, Instrument response correction, Kalman filter, Space applications, Temperature dependence.

\section{INTRODUCTION}

$\mathbf{T}$ HE NASA InSight mission will land on Mars in November 2018 and place two three-axis seismometers on the Martian surface [1]. These co-located sensors will provide valuable data for the understanding of the internal structure and geophysical processes at play on Mars. Our MEMS microseismometer is implemented as the short period sensor for the mission, however, it has been demonstrated to have a broad-band performance with a sub nano-g noise floor [2] [3] [4]. The capabilities for deployment are limited, due to the constrained power and mass. The sensor will be deployed directly onto the surface of Mars without the vault typical for terrestrial applications. Power constraints and the large Mars ambient diurnal temperature range prevent active thermal control (e.g. [5] [6]). To this end, a wind and thermal shield (WTS) will be placed on top of the seismometer to attenuate the wind, pressure and thermal contribution to the signal [7] [8] [9]. Further attenuation is provided by the packaging of the microseismometer. Within the sensor, a silicon/solder bilayer has been incorporated into the suspension to counteract

A. E. Stott, C. Charalambous and W. T. Pike are with the Department of Electrical and Electronic Engineering, Imperial College London, London, SW7 2AZ (email: alexander.stott10, constantinos.charalambous05, w.t.pike@imperial.ac.uk).

T. Warren is with the Department Atmospheric, Oceanic and Planetary Physics, Oxford University, Oxford, OX1 3PU (email: tristram.warren@physics.ox.ac.uk). the proportional change in acceleration due to temperature [10]. The aim of the techniques presented in this paper is to provide a "virtual vault" to mitigate the limitations of these passive methods. We have developed a two-step general approach that helps solve for an accurate instrument response characterisation followed by the decorrelation of a temperature dependent component from the microseismometer outputs using a physics-based model.

The first step is to determine the acceleration response of the microseismometer. This is the problem of transfer-function inversion, that is, converting the electronic velocity and mass position outputs of the instrument to physical units. This is a common task for seismic instruments and such functionality is implemented in the commonly used Seismic Analysis Code (SAC) [11]. The algorithm implemented is based on frequency division which is known to be ill-posed as the velocity and mass position channels typically have a transfer-function that tends to zero at some point [12] [13], the velocity at low frequency and the mass position at high frequency. To counteract this, seismologists will usually taper the result for each channel to obtain a corrected response in the bandwidth they are interested in [14]. However, to characterise the response of the microseismometer it is desirable to obtain the instrument's acceleration over the full available bandwidth. To this end, we propose a sensor fusion based method to combine the mass position and velocity outputs using a Kalman Filter [15]. This enables the complementary properties of the two outputs to be synthesised, producing an acceleration signal response for the microseismometer across the entire signal bandwidth. The Kalman Filter is often used in such sensor fusion applications [16] [17] but, to our knowledge, such an approach has not been used before for the outputs of a seismometer.

Next, a thermal model coupling the measured temperature signals to the acceleration response of the microseismeter is developed using the corrected signal. We incorporate the model into the Kalman Filter approach to give an improved estimation of the outputs of the sensor. This, in turn, leads to an algorithm for temperature decorrelation from the microseismometer's output. The result yields the purely seismic component measured by the microseismometer, which can then be used for the required science goals of the mission [1]. Our developed approach is physics-based, on the low-level response of the sensor across the full bandwidth.

This full-band decorrelation approach involves all the available information and is therefore limited by the physics model rather than the priors or linearisation used in other approaches 
[15]. The resulting output is valid over the full bandwidth, without processing artefacts, which is of particular value where signals of interest are not always known before deployment. For example, icy worlds like Europa present a scientific challenge where the distribution of information across the frequency spectrum is not well constrained [18]. Through applying this method to experimental data, we validate our approach and are able to quantify:

1) the contribution of our passive bilayer thermoelastic compensation;

2) the noise floor of the MEMS microseismometer out to tidal frequencies;

3) the performance down to $-65 \mathrm{C}$.

This paper will first outline the motivation behind the developments and introduce the theory of the microseismometer output channels and the Kalman filter. A method to obtain an accurate, transfer-function corrected, acceleration signal is then presented. This technique is further developed in order to determine the temperature response of the sensor. We then provide experimental results for the proposed transferfunction correction and show the performance increase available from the temperature decorrelation. The significance of the temperature decorrelation method is demonstrated through the observation of the Earth tide and the validation of the thermal compensation included in the microseismometer. We then provide an analysis of a "cold test" to prove the sensor performance in dynamic, inhospitable environments.

\section{MiCROSEISMOMETER DESCRIPTION}

\section{A. Seismic sensor instrument response and outputs}

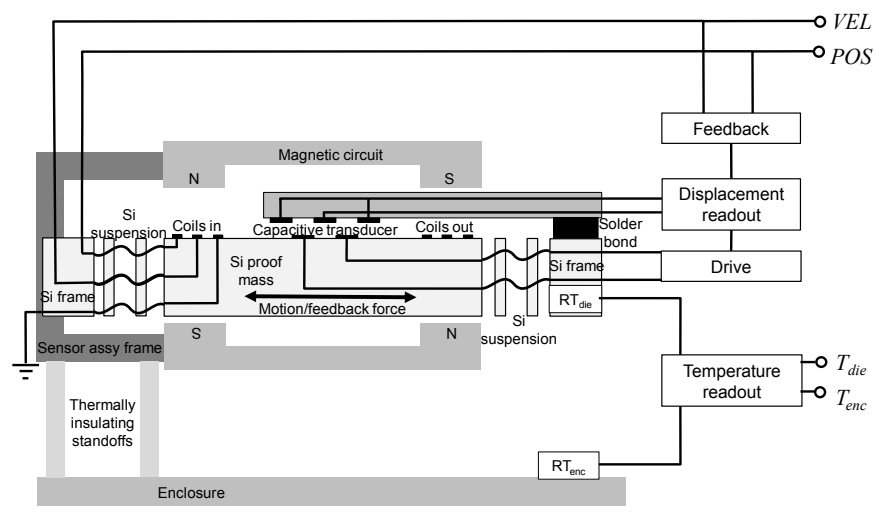

Figure 1: The schematic shows (in cross-section) the MEMS sensor, connectivity to the functional blocks of the electronics and the packaging of the sensor in its enclosure. $R T_{e n c}$ and $R T_{d i e}$ indicate the locations of the resistance thermometers.

Figure 1 shows a system diagram of the microseismometer. The sensor operates in a feedback loop where the main and integral coils are driven to oppose the force on the sensor mass, according to the force balance principle [2] [19]. The corresponding signals which drive these coils, velocity (VEL) and mass position (POS), are recorded as the outputs of the sensor. The velocity channel provides the main science data channel for the mission and its transfer-function is modelled as a two pole-zero pair high-pass filter with a corner frequency at
$f_{S P}=1 / 35 \mathrm{~Hz}$. On the other hand, the mass position channel is directly proportional to acceleration at low frequencies. The transfer-function for acceleration is well modelled by a pole-zero pair low pass filter with a corner frequency at $f_{S P}=1 / 35 \mathrm{~Hz}$. Figure 2 shows a comparison of the respective transfer-functions of the velocity and mass position channels for acceleration.

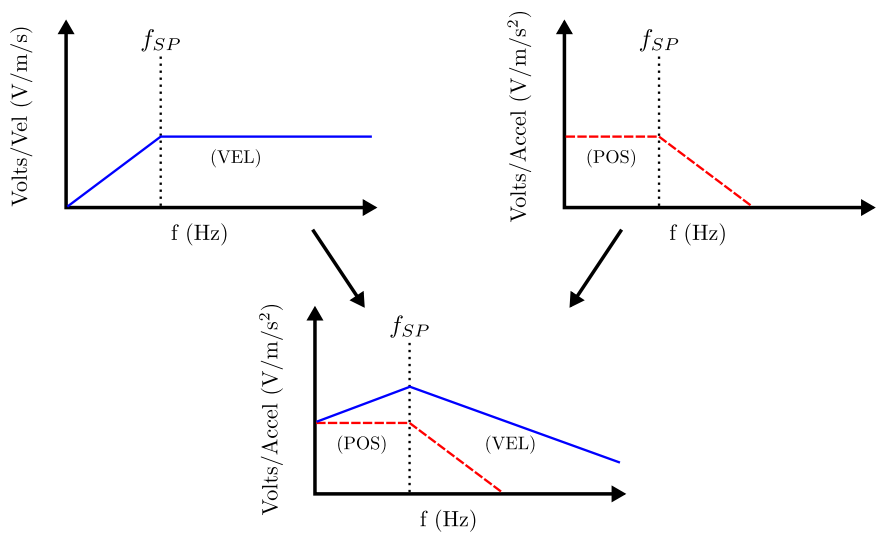

Figure 2: The top two plots show the frequency response of the velocity channel $(\mathrm{V} / \mathrm{m} / \mathrm{s})$ and mass position channel $\left(\mathrm{V} / \mathrm{m} / \mathrm{s}^{2}\right)$. The bottom plot shows both the responses to acceleration $\left(\mathrm{V} / \mathrm{m} / \mathrm{s}^{2}\right)$.

\section{B. The microseismometer thermal model}

It has been shown that a temperature input to the microseismometer produces a proportional acceleration response owing to the change in the Young's modulus of the silicon cantilever [10]. Moreover, the sensor can tilt relative to its enclosure due to the thermal response of the mounting.

The microseismometer is mounted inside an enclosure as shown in Figure 1. This housing is designed to isolate the sensor from external temperatures, that is, an externally applied temperature is not coupled directly to the sensor die. This is described by the heat equation expressed through the electrical analogy for heat transfer

$$
T_{\text {die }}=T_{\text {enc }}-R C \frac{d T_{\text {die }}}{d t}
$$

where $T_{\text {die }}$ is the temperature of the sensor die, $T_{e n c}$ is the temperature at the enclosure and $R C$ is the "time constant" of the microseismometer mounting.

The resultant temperature difference between the circuit nodes causes a heterogeneous expansion in the materials, thus tilting the sensor. As a result, the added tilt acceleration is modelled as being proportional to the difference between $T_{\text {die }}$ and $T_{\text {enc }}$. The thermal response model of the sensor is then

$$
a_{\mu S T}=\alpha_{\text {die }} T_{\text {die }}+\alpha_{\Delta} \Delta T
$$

where $a_{\mu S T}$ is the acceleration response of the microseismometer due to temperature, $\alpha_{d i e}$ is the directly proportional sensitivity to the die temperature $T_{d i e}, \alpha_{\Delta}$ is the sensitivity to the mounting temperature difference $\Delta T=T_{\text {die }}-T_{\text {enc }}$.

\section{ANALYSIS BACKGROUND}

\section{A. Coherence Noise Test}

The sensitivity of a seismometer is set by the intrinsic, or self-noise of the instrument. This is achieved through the co- 
herence noise test ${ }^{1}$ [21]. This test forms an experimental basis to obtain the instrument sensing limit, its transfer-function and its response to non-seismic factors such as temperature. A seismometer noise analysis background is found in [22] [23].

Holcomb [21] introduced a method to determine the "selfnoise" of a seismic sensor, that is, the recorded signal produced by the instrument/sensing limit of a seismometer. The seismometer at test is co-located with a reference seismometer that is assumed to have superior performance to it. The sensor noise power spectral density (PSD) is then determined through the equation

$$
\hat{P}_{N 1 N 1}=P_{X X}\left(1-\gamma^{2}\right)
$$

where $\hat{P}_{N 1 N 1}$ is an estimate of the sensor noise PSD, $P_{X X}$ is the PSD of the data obtained from the test seismometer and $\gamma$ is the spectral coherence between the reference and the test seismometer.

The seismometers' axes must be aligned to accurately calculate the coherence between them, and therefore the noise. This is especially important for the microseismometer as it is designed for operation in Martian gravity. As such, the vertical sensor must be tilted to an angle that gives an incident gravity acceleration of $3.71 \mathrm{~ms}^{-2}$. This means that a composite axis must be derived from a three-axis reference seismometer prior to the calculation in (3).

When a reference seismometer is present, we are also able to obtain the delta noise, $\Delta a$, which is defined as

$$
\Delta a=a_{\mu S}-a_{R E F}
$$

where $a_{\mu S}$ is the acceleration response derived from the outputs of the microseismometer and $a_{R E F}$ is the acceleration response derived from the outputs of the reference seismometer. This signal is useful for determining the test instruments' response to external factors. Although it is a useful approximation of the sensor's noise time series, it is not as robust as the coherence method in (3) over the full signal bandwidth. This is because both seismometer's transferfunctions must be known to an accuracy better than the ratio of the ambient signal to the self-noise, a factor of up to 1000.

\section{B. Seismometer transfer-function correction}

The frequency response of a seismometer's outputs and the corresponding correction is of interest in order to obtain a good scientific interpretation, especially for long period signals [12]. The problem is described as

$$
\begin{aligned}
y(t) & =h(t) * x(t) \\
Y(f) & =H(f) X(f)
\end{aligned}
$$

where $*$ indicates convolution, the seismic signal is denoted as $x(t)$ (Fourier transform $X(f)$ ), the output from the seismometer is given as $y(t)$ (Fourier transform $Y(f)$ ) and $h(t)$ (Fourier transform $H(f))$ is the transfer-function of the seismometer. The goal is to remove the transfer-function, $h(t)$, from the measured signal, $y(t)$, to make available the seismic signal,

\footnotetext{
${ }^{1}$ The three-sensor test of Sleeman [20] is also commonly used, however, we do not implement it here.
}

$x(t)$, for analysis. As a result, the transfer-function $h(t)$ or $H(f)$ must be known.

For many science applications, the transfer-function must be inverted. Such an operation is available in commonly used seismology toolboxes such as the seismic analysis code (SAC) [11] [14]. In such toolboxes the impact of the transfer-function is removed through a division by an estimated response in the frequency-domain. The result for velocity signals is tapered at low frequencies. This is because the theoretical transferfunction of a seismometer's outputs often tend to zero at high or low frequencies, [12] causing the inversion to be numerically ill-posed and, therefore, contain spurious results due to the required regularisation. This is the case for the VEL and POS outputs (VEL at low frequency and POS at high frequency) of the microseismometer described in Section II-A.

We propose a sensor fusion based method for transferfunction correction to combat the numerical issues of the conventional technique described above. The differentiated velocity channel provides a high fidelity acceleration signal but for the lower frequencies the output is fused with that of the mass position which provides a suitable proxy for acceleration at this scale. This method takes advantage of the complementary properties of each channel to counteract the numerical intractability from the frequency division deconvolution. As a result, a fusion of the channels will provide an accurate acceleration signal for the full bandwidth of the data. This sensor fusion is achieved using a Kalman filter [16].

\section{The Kalman Filter}

Many physical systems are described by the state-space equation

$$
\mathbf{x}_{k}=\mathbf{F} \mathbf{x}_{k-1}+\mathbf{B} \mathbf{u}_{k}+\mathbf{w}_{k}
$$

where $\mathbf{x}_{k}$ is the state vector containing the value of interest for the system at time instant $k$, the vector $\mathbf{u}_{k}$ are the inputs to the system related to the state vector through the matrix $\mathbf{B}$, $\mathbf{w}_{k}$ is an additive noise term and the matrix $\mathbf{F}$ describes the forward evolution of the state vector from the previous time instant $k-1$. There is sometimes uncertainty in the accuracy of the forward model either through missing physics or noisy inputs. This is accounted for by the noise vector $\mathbf{w}_{k}$ which has the covariance matrix $\mathbf{Q}$. Such a system can also be measured through the equation

$$
\mathbf{z}_{k}=\mathbf{H} \mathbf{x}_{k}+\mathbf{v}_{k}
$$

where $\mathbf{z}_{k}$ is the vector of measurements and the matrix $\mathbf{H}$ defines the relation between the measurements and the state vector, $\mathbf{x}_{k}$. The vector $\mathbf{v}_{k}$ is an additive noise term which accounts for uncertainty in the measurement equation and has covariance matrix $\mathbf{R}$. Owing to the uncertainty in each equation, the state vector $\mathbf{x}_{k}$ from the forward model (6) may differ to that derived from the measurement equation (7). The covariance matrix of the error between the state vector and its estimate is denoted as $\mathbf{P}_{k \mid k-1}=E\left[\left(\mathbf{x}_{k}-\hat{\mathbf{x}}_{k \mid k-1}\right)\left(\mathbf{x}_{k}-\right.\right.$ $\left.\left.\hat{\mathbf{x}}_{k \mid k-1}\right)^{\mathrm{T}}\right]$ for the a priori prediction from the previous time step $\hat{\mathbf{x}}_{k \mid k-1}$ and $\mathbf{P}_{k \mid k}=E\left[\left(\mathbf{x}_{k}-\hat{\mathbf{x}}_{k \mid k}\right)\left(\mathbf{x}_{k}-\hat{\mathbf{x}}_{k \mid k}\right)^{\top}\right]$ for the a posteriori estimate $\hat{\mathbf{x}}_{k \mid k}$. 
The Kalman filter, summarised in Algorithm 1, solves this problem to produce an optimal estimate of the state of a system described by two such equations. This is achieved through the Kalman gain, $\mathbf{K}$, which mixes the predictions derived from the measurements and the forward model. The mixture depends on the level of uncertainty of each model, described through the noise covariance matrices $\mathbf{Q}$ and $\mathbf{R}$. As a result, the Kalman gain calculates how much to "trust" either the measurement or forward model. The combination is then given as the optimal prediction of the state vector $\mathbf{x}_{k}$. A detailed derivation can be found in [15] [24].

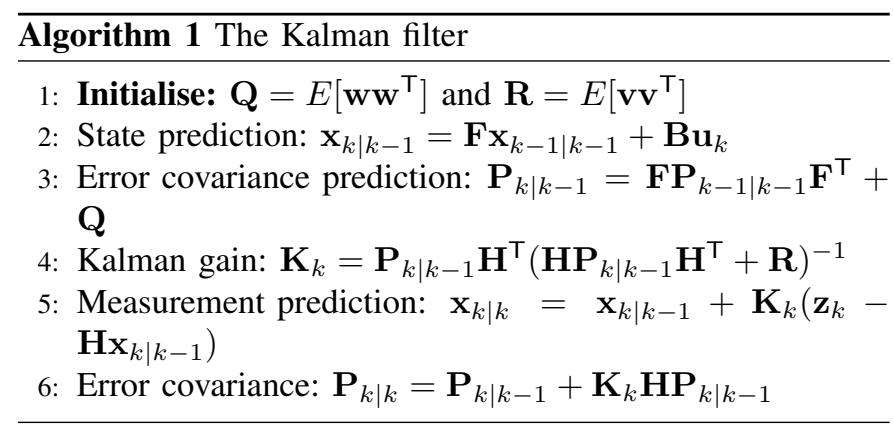

1) The Indirect Kalman Filter: The Kalman filter has been introduced here as a method to estimate the state values of a system based on both a physical model and measurement data. The filter can be implemented in an "indirect" form, where the state vector estimates a hidden or error state [25].

In many applications it is known that a particular sensor has an inherent instability or drift and so its data are not suitable as a state value over the full frequency range. Consequently, this indirect Kalman filter topology is frequently used for obtaining high quality measurements from inertial sensors [16] [26].

\section{Full-Band Sensor Fusion Algorithms}

\section{A. Sensor fusion for transfer-function correction}

In order to obtain an acceleration signal from the microseismometer for all frequencies we synthesise the acceleration response derived from the transfer-function corrected velocity channel and the mass position output. This is achieved by implementing a Kalman filter in an indirect topology where the error state is the error in the microseismometer acceleration signal derived from the velocity channel. In this case we view the acceleration signal derived from the velocity output as a stable measurement containing an erroneous low frequency component, whereas the mass position output provides a good low frequency measurement of the acceleration. To this end, we construct the state variable

$$
\mathbf{x}_{k}=\left[\begin{array}{l}
a_{\mu S, k} \\
a_{e r r, k}
\end{array}\right]
$$

where $k$ is the time instant, $a_{\mu S, k}$ denotes the acceleration response of the microseismometer and $a_{e r r, k}$ is the artefact introduced as a result of the ill-posed transfer-function correction of the velocity channel. The state equation (6) is then given

$$
\mathbf{F}=\left[\begin{array}{cc}
0 & -1 \\
0 & 1
\end{array}\right] \quad \mathbf{B}=\left[\begin{array}{l}
1 \\
0
\end{array}\right]
$$

where the input is

$$
\mathbf{u}_{k}=a_{V E L, k}
$$

with $a_{V E L}$ denoting the acceleration signal derived from the velocity channel. The measurement equation (7) is then given

$$
\mathbf{z}_{k}=a_{P O S, k} \quad \mathbf{H}=\left[\begin{array}{ll}
1 & 0
\end{array}\right]
$$

where $a_{P O S}$ is the acceleration derived from the mass position.

At each time instant, $k$, the prediction of $a_{\mu S, k}$ from the forward model (Step 2 in Algorithm 1) is given as $a_{V E L, k}-a_{e r r, k-1}$. This result (along with the estimate of $\left.a_{e r r, k}\right)$ is adjusted by the error to the "measurement", which is the acceleration derived from the mass position, $a_{P O S}$, in Step 5 of Algorithm 1. The level of the adjustment is dictated by the Kalman gain, $\mathbf{K}_{k}$, which is calculated using the noise covariance matrices for the forward model, $\mathbf{Q}$, and the measurement, $\mathbf{R}$. In this indirect topology the respective noise power assigns the relative level of "trust" to each equation. As such, the covariance matrix choice dictates the mixture of $a_{V E L}$ and $a_{P O S}$ in producing the estimate of the state variable $\mathbf{x}_{k}=\left[a_{\mu S, k}, a_{e r r, k}\right]^{\top}$, demonstrated in Section V-B. Therefore, the computation of the state vector is recursive and this implementation operates as a filter.

Remark 1. The Kalman filter for transfer-function correction (K-TF). An accurate acceleration response from the microseismometer is obtained by applying the Kalman Filter in Algorithm 1 with chosen noise covariance matrices, $\mathbf{Q}$ and $\mathbf{R}$, to the system described in (8), (9), (10) and (11). This is the Kalman filter for transfer-function correction $(K-T F)$.

\section{B. Full-band temperature decorrelation}

The thermal model for the microseismometer acceleration response, (2), can be seen as a measurement of the acceleration itself. In fact, the thermal response dominates the signal at low frequencies. This can therefore be added to the Kalman filter model outlined in Section IV-A to provide an improved estimate of the acceleration along with the mass position. The Kalman filter topology now becomes

$$
\begin{gathered}
{\left[\begin{array}{c}
a_{\mu S, k} \\
a_{\text {err }, k}
\end{array}\right]=\left[\begin{array}{cc}
0 & -1 \\
0 & 1
\end{array}\right]\left[\begin{array}{c}
a_{\mu S, k-1} \\
a_{e r r, k-1}
\end{array}\right]+\left[\begin{array}{l}
1 \\
0
\end{array}\right] a_{V E L, k}+\mathbf{w}_{k}} \\
{\left[\begin{array}{c}
T_{\text {die }, k} \\
\Delta T_{k} \\
a_{P O S, k}
\end{array}\right]=\left[\begin{array}{ccc}
\frac{\alpha_{\text {die }}}{2} & \frac{\alpha_{\Delta}}{2} & \frac{1}{2} \\
0 & 0 & 0
\end{array}\right]^{+}\left[\begin{array}{l}
a_{\mu S, k} \\
a_{e r r, k}
\end{array}\right]+\mathbf{v}_{k}}
\end{gathered}
$$

where $(\cdot)^{+}$denotes the generalised inverse. The measurements now include the die temperature and temperature difference as well as the mass position. The coefficients are given from an initial regression and are scaled by a factor of two to average the information included in both measurements.

Remark 2. The Kalman filter for acceleration fusion from mass position and temperature $(K-T F+T)$. The thermal model for the microseismometer acceleration in (2) can be incorporated as a measurement into the system outlined by (12) and (13). Applying the Kalman filter in Algorithm 1 to this system yields an estimation of the acceleration response, $a_{\mu S}$, from the sensor. 
Table I: Datasets used

\begin{tabular}{|c|c|c|c|c|c|c|c|c|}
\hline Name & Location & Reference & Length & $\begin{array}{l}\text { Temperature } \\
\text { rate }(\mathrm{Hz})\end{array}$ & $\begin{array}{l}\text { Filter Stop- } \\
\text { Band frequency } \\
(\mathrm{Hz})\end{array}$ & $\begin{array}{l}\text { Temperature } \\
\text { variance }\end{array}$ & & Notes \\
\hline QM Vert 2017 & Oxford & $\begin{array}{l}\text { Guralp } \\
\text { CMG-3T }\end{array}$ & 49 hours & $1 / 30$ & $9 \times 10^{-5}$ & $\begin{array}{l}\operatorname{var}\left(T_{\text {die }}\right) \\
1.4 \times 10^{-2} \\
\operatorname{var}(\Delta T) \\
9.9 \times 10^{-6}\end{array}$ & $\begin{array}{l}= \\
=\end{array}$ & $\begin{array}{l}\text { No solder-silicon bilayer thermal com- } \\
\text { pensation, ambient temperature noise } \\
\text { test }\end{array}$ \\
\hline QM Vert 2016 & Oxford & $\begin{array}{l}\text { Guralp } \\
\text { CMG-3T }\end{array}$ & 112 hours & $1 / 60$ & $2 \times 10^{-5}$ & $\begin{array}{l}\operatorname{var}\left(T_{\text {die }}\right) \\
2.1 \times 10^{-3} \\
\operatorname{var}(\Delta T) \\
1.6 E-6\end{array}$ & $\begin{array}{l}= \\
=\end{array}$ & $\begin{array}{l}\text { No solder-silicon bilayer thermal com- } \\
\text { pensation, ambient temperature noise } \\
\text { test }\end{array}$ \\
\hline QM Vert Cold & $\begin{array}{l}\text { Oxford Ther- } \\
\text { mal Vacuum }\end{array}$ & $\begin{array}{l}\text { Guralp } \\
\text { CMG-3T }\end{array}$ & 49 hours & $1 / 60$ & $2 \times 10^{-4}$ & $\begin{array}{l}\operatorname{var}\left(T_{\text {die }}\right) \\
462 \\
\operatorname{var}(\Delta T) \\
2.5 \times 10^{-3}\end{array}$ & $\begin{array}{l}= \\
=\end{array}$ & $\begin{array}{l}\text { Performed in a cold testing rig, no } \\
\text { solder-silicon bilayer thermal compen- } \\
\text { sation, low temperature noise test }\end{array}$ \\
\hline FM Vert & CNES & None & 6.4 hours & $1 / 5$ & $7 \times 10^{-3}$ & $\begin{array}{l}\operatorname{var}\left(T_{d i e}\right) \\
5.3 \\
\operatorname{var}(\Delta T) \\
9.8 E-2\end{array}$ & $\begin{array}{l}= \\
=\end{array}$ & $\begin{array}{l}\text { FM signal chain, thermal sensitivity } \\
\text { test }\end{array}$ \\
\hline FM Horiz & CNES & None & 4.4 hours & $1 / 5$ & $7 \times 10^{-3}$ & $\begin{array}{l}\operatorname{var}\left(T_{\text {die }}\right) \\
11.5 \\
\operatorname{var}(\Delta T) \\
0.34\end{array}$ & $\begin{array}{l}= \\
=\end{array}$ & $\begin{array}{l}\text { FM signal chain, thermal sensitivity } \\
\text { test }\end{array}$ \\
\hline
\end{tabular}

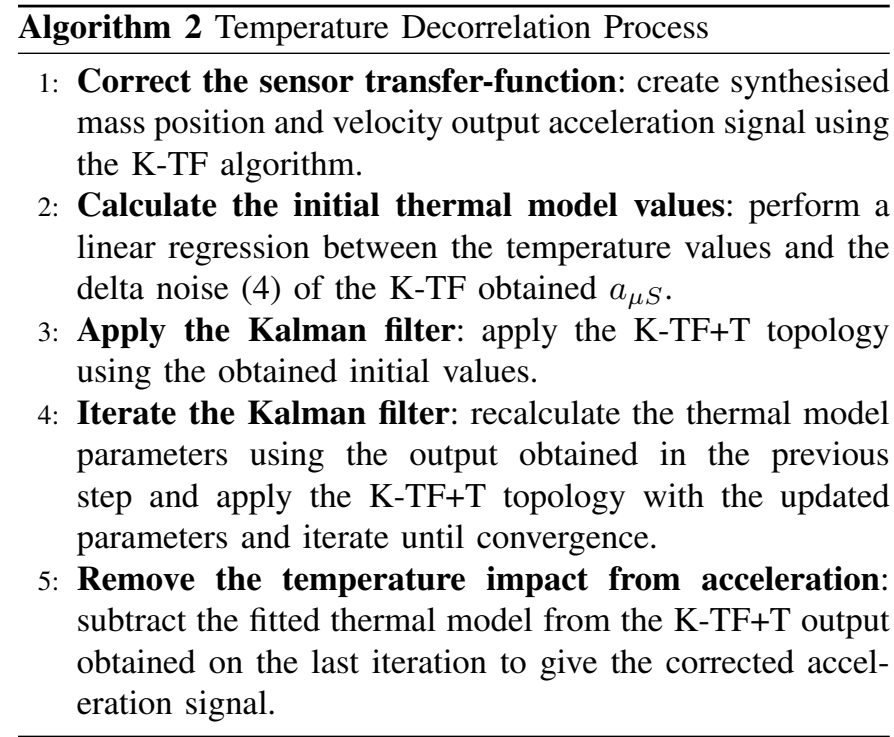

This evolution of the K-TF can be used to provide both a refined estimation of the thermal model parameters in (2) and a decorrelation of the thermally induced acceleration from the sensor output. To this end, we introduce Algorithm 2. The estimate of the microseismometer acceleration response, $a_{\mu S}$, is given as the output of the final iteration of the K-TF+T method in Step 4. The parameters for the thermal model (2) are calculated through a regression to the delta noise (4) to give an estimate of the temperature dependent microseismometer acceleration, $a_{\mu S T}$. The temperature decorrelated acceleration response is then given as $a_{\mu S}-a_{\mu S T}$. This novel procedure allows us to better determine the performance of the microseismometer in relation to its thermal response (including determining the effect of the thermal compensation [10]) and then show the sensor's intrinsic noise at low frequencies. Details on the implementation of the K-TF and K-TF+T methods for Algorithm 2 are provided in Appendix A.

\section{EXPERIMENTAL RESULTS}

\section{A. Experimental setup}

Experimental data has been obtained from testing in two locations: Oxford University and CNES in Toulouse. The data obtained from Oxford are from a qualification model (QM) vertical sensor and the data obtained from CNES are of vertical and horizontal flight model (FM) sensors that will be flown on the InSight mission. In total five datasets were used to demonstrate the proposed $\mathrm{K}-\mathrm{TF}$ and $\mathrm{K}-\mathrm{TF}+\mathrm{T}$ algorithms:

- two ambient temperature noise tests performed in a seismically quiet laboratory at Oxford which we refer to as QM Vert 2017 and QM Vert 2016;

- two temperature sensitivity tests in the CNES cleanroom which we refer to as FM Vert and FM Horiz;

- a low temperature test at Oxford in a specially designed "cold test" facility which we refer to as QM Vert Cold.

Table I summarises the collected data. In all cases the data were decimated or interpolated from their original acquisition rate to a sampling frequency of $10 \mathrm{~Hz}$. Furthermore, all the signals were zero-meaned prior to analysis but no additional detrending was performed. The equipment and nature of the different test facilities is described in the following.

1) Ambient temperature noise test setup: The two ambient temperature tests were performed in the basement of the Atmospheric, Ocean and Planetary Physics building. A vertical microseismometer was placed in a test chamber and tilted so the gravity vector along the sensor axis was equivalent to Martian gravity. This vertical sensor did not have the thermal compensation described in [10]. The velocity and mass position outputs were then sampled at $100 \mathrm{~Hz}$ by a 24bit Centaur data logger. A Guralp three-axis seismometer was recorded alongside the microseismometer with the same data logger. This was then used to obtain an estimate of the sensor's self-noise through the coherence test (3).

2) Low temperature noise test setup: The cold test of the $\mathrm{QM}$ vertical unit was performed in a specialist rig designed to 


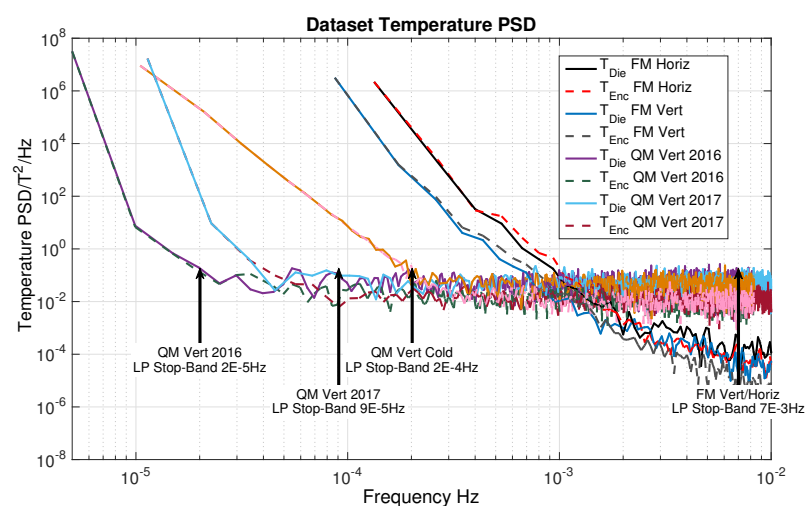

Figure 3: PSD of the thermometer temperatures in each dataset and their respective low-pass filter stop-band frequencies. The two FM datasets have a lower acquisition noise floor.

determine the microseismometer's behaviour down to typical Mars temperatures [27]. The inside of the rig was cooled by a liquid nitrogen system which was then allowed to passively warm. Inside the rig, the vertical microseismometer was installed and recorded in the same way as the ambient temperature tests. A Guralp reference seismometer was placed outside the rig but on a coupled platform to the microseismometer mount in order to record the ambient seismic signal and enable a coherence test (3).

3) Temperature sensitivity test setup: Experiments were performed on the FM units at CNES with the specific goal to determine the temperature sensitivity of the sensor. These were done in a cleanroom facility and the data were recorded through the flight data logger, the "Ebox". The Ebox considers a channel to either be a "science" or "housekeeping" (HK) output. The microseismometer velocity signal is considered to be a science output and so is sampled at $100 \mathrm{~Hz}$ and quantised on a 24-bit ADC. However, the mass position is considered to be a housekeeping signal and so are sampled at $1 \mathrm{~Hz}$ and quantised with a 12-bit ADC. The sensor mounting was attached to a gonionmeter which tilted the vertical microseismometer to simulate Martian gravity. No tilting was required for the testing of the horizontal microseismometer. No reference seismometer was available to allow for a self-noise determination. The sensor assembly was externally heated from $\sim 20 \mathrm{C}$ to $\sim 30 \mathrm{C}$ and allowed to cool passively.

4) Temperature data acquisition: The $T_{\text {enc }}$ and $T_{\text {die }}$ temperature signals were measured respectively by a platinum resistance thermometer (PRT) and a gold resistance thermometer, integrated in the sensor die itself, and recorded on a 24-bit data logger. The resistance values were converted to temperature and low-pass filtered with an IIR filter designed to remove the contribution of the measurement noise. In each case the filter was designed to have $15 \mathrm{~dB}$ attenuation between the stop-band frequency (given in Table I) and the pass-band frequency, which was $1.1 \times 10^{-1} \mathrm{~Hz}$ below. The filters had a pass-band ripple of $1 \mathrm{~dB}$ and an attenuation of $40 \mathrm{~dB} / \mathrm{decade}$ above the stop-band frequency. This stop-band frequency was determined through inspection of the temperature PSDs in Figure 3.
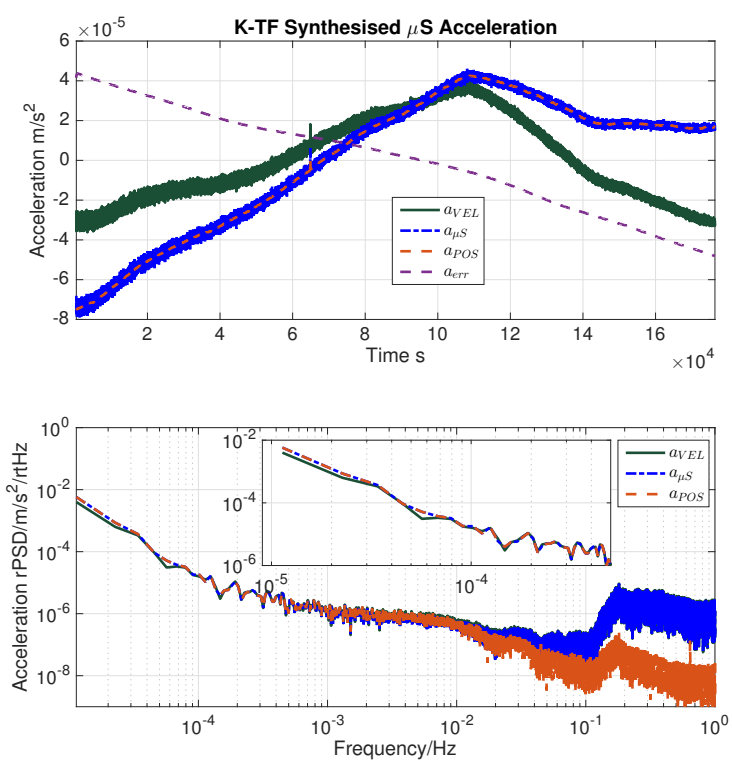

Figure 4: K-TF synthesised microseismometer acceleration, $a_{\mu S}$, time series and rPSD compared to $a_{V E L}$ and $a_{P O S}$ for QM Vert 2017 dataset.

\section{B. Mass position and velocity output fusion}

The K-TF method for transfer-function inversion in Remark 1 was applied to the QM Vert 2017 dataset. The velocity channel output time series was corrected using the standard ill-posed frequency division algorithm described in Section III-B and converted to an acceleration signal, $a_{V E L}$ in (10). The mass position output was purely scaled by a gain factor to convert it to an acceleration signal, $a_{P O S}$ in (11), as the response to acceleration is flat in the desired bandwidth for the sensor fusion. The time series and root PSD (rPSD) of $a_{V E L}$, $a_{P O S}$ and the sensor fusion result of the K-TF method, $a_{\mu S}$, are shown in Figure 4. In the time domain, the synthesised acceleration response, $a_{\mu S}$, is similar to the temperature profile (in the fourth pane of Figure 5) when compared to that derived from the velocity channel, $a_{V E L}$. Therefore, the sensor fusion for transfer-function correction has produced a physically meaningful acceleration response over the entire bandwidth. This result can then be used to obtain a corrected time series in any desired bandwidth, without any processing artefacts, for further analysis.

\section{Validation of the microseismometer thermal compensation and temperature decorrelation}

The validation of Algorithm 2 will in turn validate the SP performance at low frequencies, the effectiveness of the thermal compensation developed in [10], the microseismometer performance at extremely low temperatures and the ability of the sensor to observe tidal signals. The obtained parameters for the thermal model (2) are given in Table II, which reports the value of the coefficients with a standard error calculated as the diagonal elements of $S_{\text {error }}=\left(\left(\mathbf{X}^{\top} \mathbf{X}\right)^{-1}(\mathbf{y}-\mathbf{X b})^{2}\right)^{\frac{1}{2}}$ where $\mathbf{X}=\left[T_{\text {die }}, \Delta T, 1\right]$ is the data matrix of the thermal model components with a bias component, $\mathbf{y}=a_{\mu S T}$ is the temperature dependent microseismometer acceleration, and 

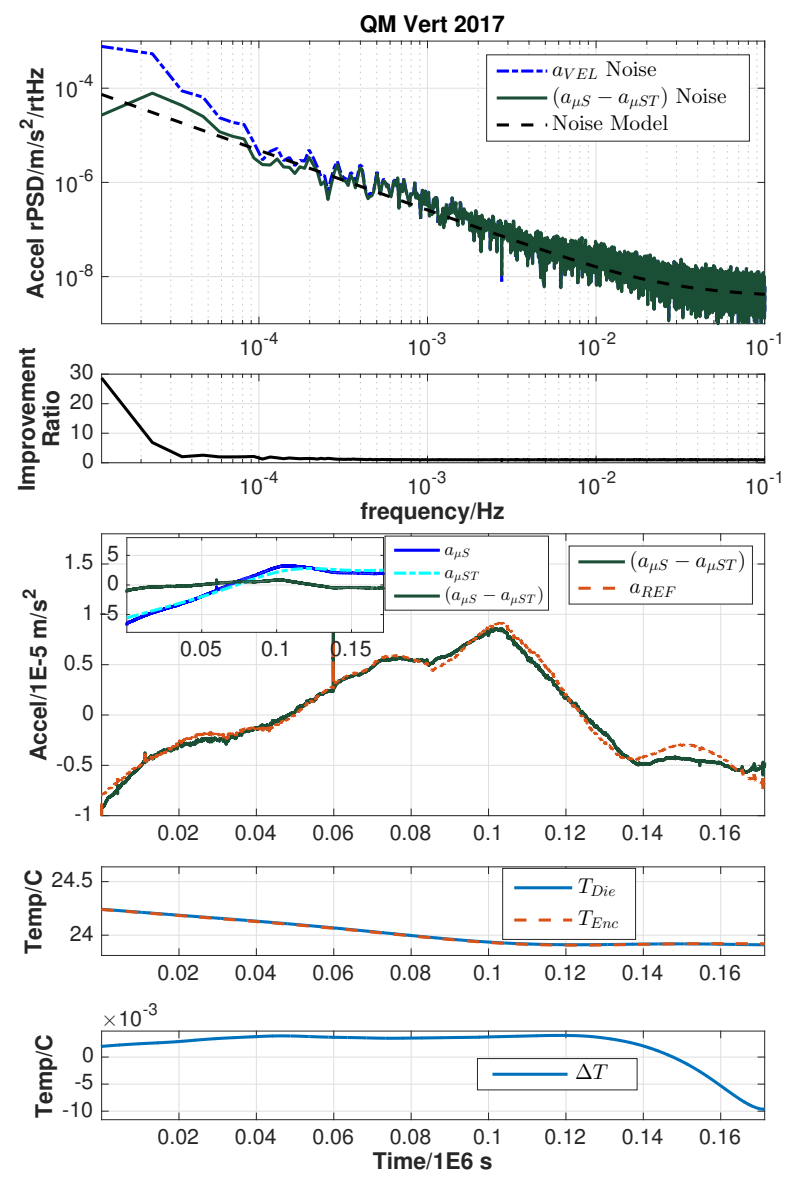

Figure 5: QM Vert 2017. First pane: Self-noise determination from the $a_{V E L}$ and temperature decorrelated, $a_{\mu S}-a_{\mu S T}$, signals. Second pane: Improvement ratio between self-noise before and after temperature decorrelation. Third Pane: acceleration time series for the reference, $a_{R E F}$, and decorrelated microseismometer acceleration with a comparison of the $\mathrm{K}-\mathrm{TF}+\mathrm{T}$ synthesised acceleration and thermal model fit inset. Fourth pane: $T_{d i e}$ and $T_{\text {enc }}$ signals, Fifth pane: $\Delta T$ signal.

$\mathbf{b}=\left[\alpha_{\text {die }}, \alpha_{\Delta}, b\right]^{\top}$ is the vector of regression coefficients. The $R^{2}$ statistic is also reported as KF1 and KF2 respectively for the fit to the K-TF produced $a_{\mu S}$ (Step 2 of Algorithm 2) and for the final iteration of the $\mathrm{K}-\mathrm{TF}+\mathrm{T}$ produced $a_{\mu S}$ (Step 4 of Algorithm 2). The derived self-noise is with respect to the Guralp reference seismometer acceleration response, $a_{R E F}$.

The process in Algorithm 2 was applied to the QM Vert 2017 dataset and the results are shown in Figure 5. The obtained linear sensitivity parameter is $\alpha_{\text {die }}=-2.50 \pm$ $0.12 \times 10^{-4} \mathrm{~ms}^{-2} / \mathrm{K}$ which is near the theoretical value of $-2.2 \times 10^{-4} \mathrm{~ms}^{-2} / \mathrm{K}$ (or $-60 \mathrm{ppm} / \mathrm{K}$ ) for an uncompensated sensor [10]. This dataset has a slowly varying temperature, this means the $\Delta T$ signal has a low variance (Table I) and is less significant to the fit than the linear parameter $\alpha_{\text {die }}$. As a result, the $\alpha_{\Delta}$ component is poorly determined, as seen by the coefficient's standard error.

Algorithm 2 was also applied to the FM units, of which the vertical microseismometer has the thermal compensation technology. In these tests no reference seismometer was available. Therefore, a purely seismic component cannot be removed from the microseismometer acceleration to calculate the Delta
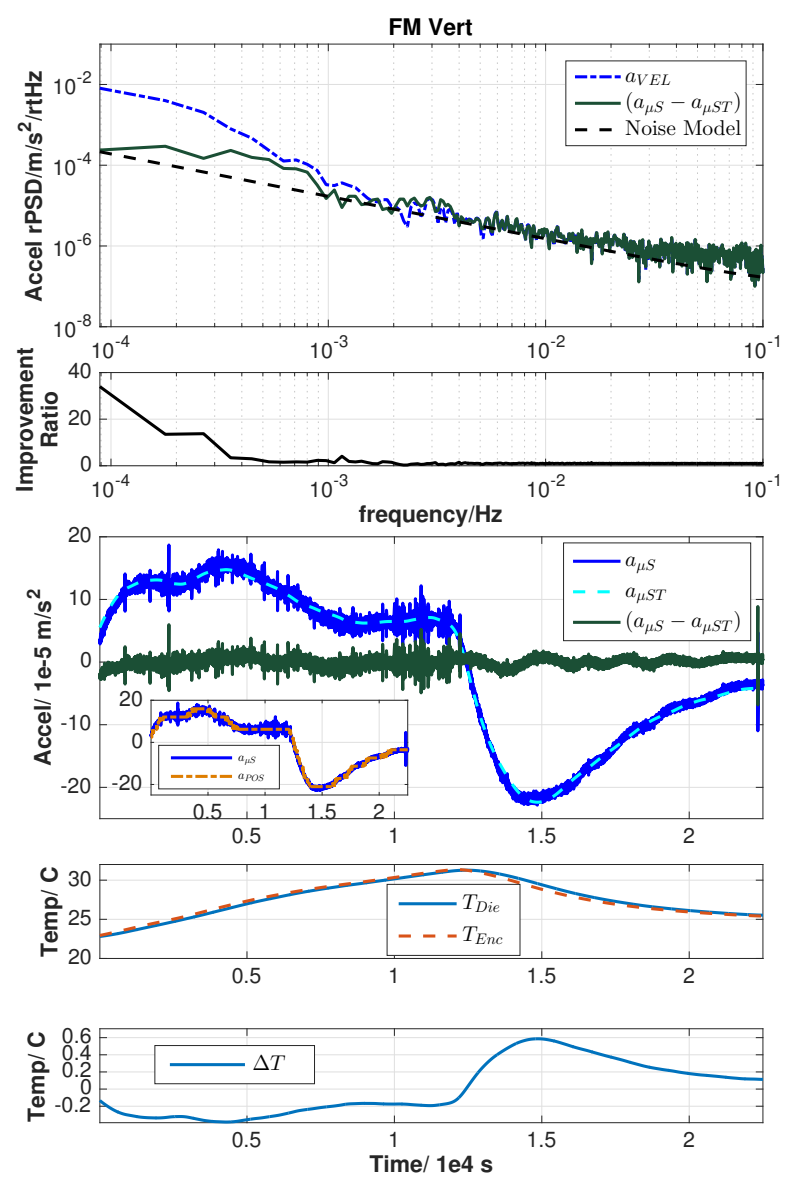

Figure 6: FM Vert. First pane: rPSD of the $a_{V E L}$, and temperature decorrelated, $a_{\mu S}-a_{\mu S T}$, signals. Second pane: Improvement ratio between rPSDs before and after temperature decorrelation. Third Pane: time series for the decorrelated microseismometer acceleration compared to the $\mathrm{K}-\mathrm{TF}+\mathrm{T}$ synthesised acceleration and thermal model fit with the $\mathrm{K}-\mathrm{TF}+\mathrm{T}$ synthesised acceleration and mass position inset. Fourth pane: $T_{d i e}$ and $T_{e n c}$ signals. Fifth pane: $\Delta T$ signal.

Noise (4), and so the temperature dependent acceleration, $a_{\mu S T}$, was approximated by the sensor output. This is a reasonable assumption as the low frequency ambient seismic signal is much less than the microseismometer's output when subjected to large temperature variation. As such, the acceleration rPSD provides a reasonable estimation of the microseismometer self-noise. The results for the FM Vert dataset are shown in Figure 6. In this case, the microseismometer acceleration is more correlated to the $\Delta T$ signal than the $T_{d i e}$ component, conversely to the QM Vert 2017 case. This is seen as the thermal model parameter $\alpha_{\Delta}=-3.80 \pm 0.21 \times 10^{-4} \mathrm{~ms}^{-2} / \mathrm{K}$ is well determined in the standard error compared to the parameter $\alpha_{\text {die }}=-0.84 \pm 2.78 \times 10^{-6} \mathrm{~ms}^{-2} / \mathrm{K}$. Therefore, the $\Delta T$ component is now more significant to the thermal model of the microseismometer's acceleration response, owing to the reduced linear sensitivity. The $\alpha_{\text {die }}$ parameter determined for the FM vertical unit is at least under two orders of magnitude lower than the uncompensated QM sensor, thus validating the performance of the thermal compensation [10]. Moreover, our approach was highly effective at decorrelating the thermal component from the signal with a reduction of up to 35 times in the low frequency seismic amplitude. The 

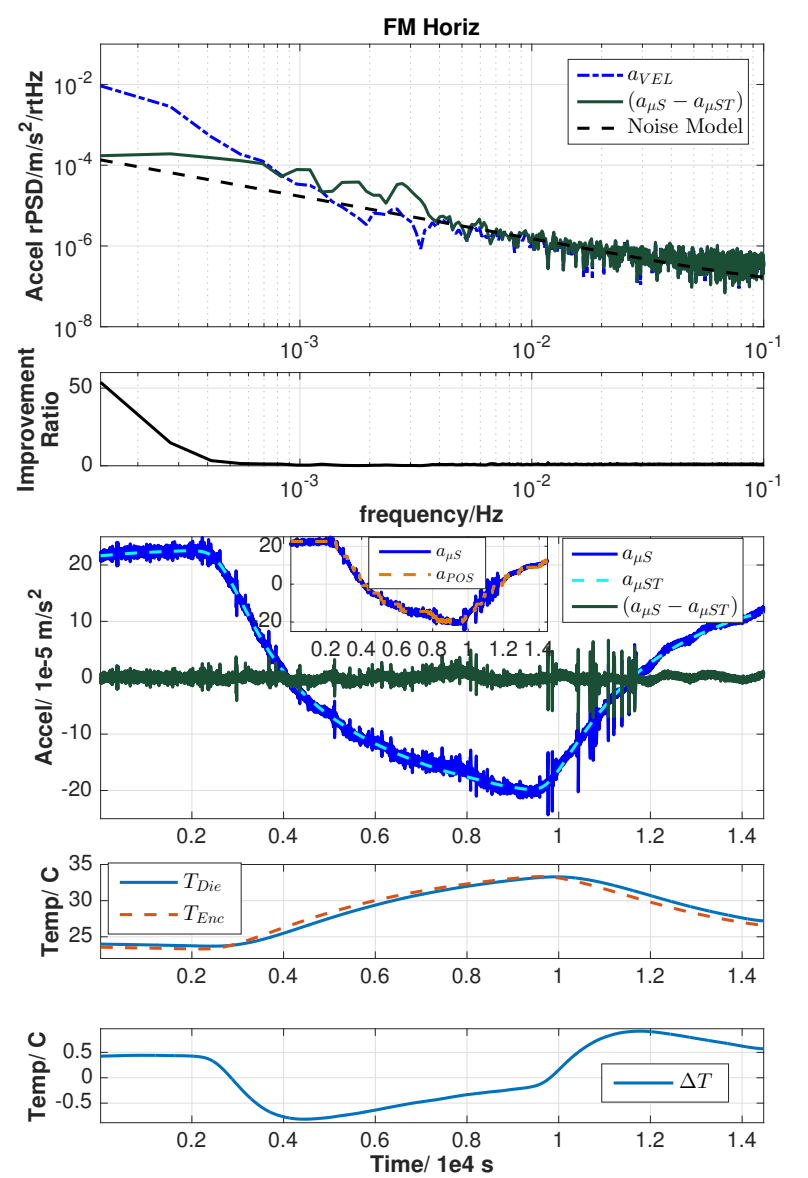

Figure 7: FM Horiz. First pane: rPSD of the $a_{V E L}$, and temperature decorrelated, $a_{\mu S}-a_{\mu S T}$, signals. Second pane: Improvement ratio between rPSDs before and after temperature decorrelation. Third Pane: time series for the decorrelated microseismometer acceleration compared to the $\mathrm{K}-\mathrm{TF}+\mathrm{T}$ synthesised acceleration and thermal model fit with the $\mathrm{K}-\mathrm{TF}+\mathrm{T}$ synthesised acceleration and mass position inset. Fourth pane: $T_{d i e}$ and $T_{e n c}$ signals. Fifth pane: $\Delta T$ signal.

rPSD of the temperature decorrelated signal, $a_{\mu S}-a_{\mu S T}$ in Figure 7, has a higher power than the $a_{V E L}$ signal around $2 \times 10^{-3} \mathrm{~Hz}$. This is because the mass position is less finely quantised in the FM electronics compared to the velocity channel, introducing an error in this bandwidth. This could be mitigated in the future by a more targeted approach to the removal of signal-correlated quantisation errors through, for example, interpolation.

On the other hand, the horizontal FM unit does not have thermal compensation as the proof mass will not be subject to an appreciable component of gravity. Only change in the tilt, $\Delta \theta$, of the sensor will produce a signal due to the change in the gravity component, giving some linear temperature sensitivity. The results for the FM Horiz dataset are shown in Figure 7 with the thermal model parameters determined as $\alpha_{\text {die }}=-3.72 \pm 0.15 \times 10^{-5} \mathrm{~ms}^{-2} / \mathrm{K}$ and $\alpha_{\Delta}=1.248 \pm 0.086 \times 10^{-4} \mathrm{~ms}^{-2} / \mathrm{K}$. The thermal signal has again been effectively decorrelated from the data with a reduction of 50 times in the low frequency seismic amplitude.

1) Tidal signals on the microseismometer: The QM Vert 2016 dataset is taken over 112 hours. As a result, it is possible to observe tidal frequencies in the record [5] [12]. However,
Table II: Thermal Model Parameters and Fit Quality

\begin{tabular}{|c|c|c|c|c|}
\hline \multirow[b]{2}{*}{ Dataset } & \multicolumn{2}{|c|}{ Thermal Model Parameter $\left(\mathrm{ms}^{-2} / \mathrm{K}\right)$} & \multicolumn{2}{|c|}{ Fit $R^{2}$} \\
\hline & $\alpha_{\text {die }}$ & $\alpha_{\Delta}$ & KF1 & $\mathrm{KF} 2$ \\
\hline QM Vert 2017 & $\begin{array}{l}-2.5 \mathrm{E}-4 \\
\pm 0.12 \mathrm{E}-4\end{array}$ & $\begin{array}{l}2.2 \mathrm{E}-4 \\
\pm 4.1 \mathrm{E}-4\end{array}$ & 0.986 & 0.998 \\
\hline QM Vert 2016 & $\begin{array}{l}-2.629 \mathrm{E}-3 \\
\pm 0.034 \mathrm{E}-3\end{array}$ & $\begin{array}{l}1.75 \mathrm{E}-2 \\
\pm 0.12 \mathrm{E}-2\end{array}$ & 0.999 & 1.000 \\
\hline QM Vert Cold & $\begin{array}{l}-1.080 \mathrm{E}-4 \\
\pm 0.016 \mathrm{E}-4\end{array}$ & $\begin{array}{l}-6.1 \mathrm{E}-4 \\
\pm 6.9 \mathrm{E}-4\end{array}$ & 0.999 & 1.000 \\
\hline FM Vert & $\begin{array}{l}-0.84 \mathrm{E}-6 \\
\pm 2.78 \mathrm{E}-6\end{array}$ & $\begin{array}{l}-3.80 \mathrm{E}-4 \\
\pm 0.21 \mathrm{E}-4\end{array}$ & 0.981 & 0.997 \\
\hline FM Horiz & $\begin{array}{l}-3.72 \mathrm{E}-5 \\
\pm 0.15 \mathrm{E}-5\end{array}$ & $\begin{array}{l}1.248 \mathrm{E}-4 \\
\pm 0.086 \mathrm{E}-4\end{array}$ & 0.979 & 0.999 \\
\hline
\end{tabular}

a temperature dependent signal in the data would obscure this tidal signal. Figure 8 shows the results for the temperature decorrelation method applied to the QM Vert 2016 dataset. The decorrelated time series contains a significant component also seen by the reference seismometer at the M2 tidal frequency, which is not observable in the uncorrected time series in the inset figure. Moreover, the self-noise estimate of the sensor now continues to follow the power law seen up to $1 \times 10^{-5} \mathrm{~Hz}$ with an improvement ratio of up to 28 . This demonstrates the ability of Algorithm 2 to recover physical signals accurately and so provide a seismometer output for a wide range of science applications.

The overall temperature variation in this experiment is the lowest of the datasets, as seen by the variance of $T_{d i e}$ and $\Delta T$ (in Table I). Due to this low variation, the temperature of each part of the system was coupled to $T_{d i e}$. Therefore, $\alpha_{\Delta}$ does not contribute significantly to the acceleration response and so is poorly determined. Furthermore, the obtained $\alpha_{\text {die }}$ parameter is not representative of just the sensor. Specifically, the feedback electronics board used in this test was known to have a high temperature sensitivity, subsequently corrected for the QM Vert 2017 and FM tests.

2) Cold testing of the microseismometer: The results for the low temperature test are shown in Figure 9 with the thermal model parameters determined as $\alpha_{\text {die }}=$ $-1.080 \pm 0.016 \times 10^{-4} \mathrm{~ms}^{-2} / \mathrm{K}$ and $\alpha_{\Delta}=-6.1 \pm$ $6.9 \times 10^{-4} \mathrm{~ms}^{-2} / \mathrm{K}$. Again in this test the $T_{\text {die }}$ component is much more significant to the acceleration response than the $\Delta T$ signal and so $\alpha_{\Delta}$ is poorly determined. Notice that the linear sensitivity parameter, $\alpha_{\text {die }}$ is $1.42 \times 10^{-4} \mathrm{~ms}^{2} / \mathrm{K}$ greater than the $\alpha_{\text {die }}$ value obtained from the QM Vert 2017 test. This corresponds to the tilting in the cold test rig mount caused by the large temperature difference between the chamber and the ambient. As the ambient is relatively constant, the temperature difference is proportional to $T_{\text {die }}$ and so the $\alpha_{d i e}$ coefficient also accounts for its effect. The tilt from the test rig is equivalent to $1.49 \times 10^{-5} \mathrm{rad} / \mathrm{K}$, a low value indicative of the careful thermoelastic design of the cold test rig.

\section{CONCLUSION}

A sensor fusion based method for transfer-function correction using a Kalman filter has been introduced. This has been 

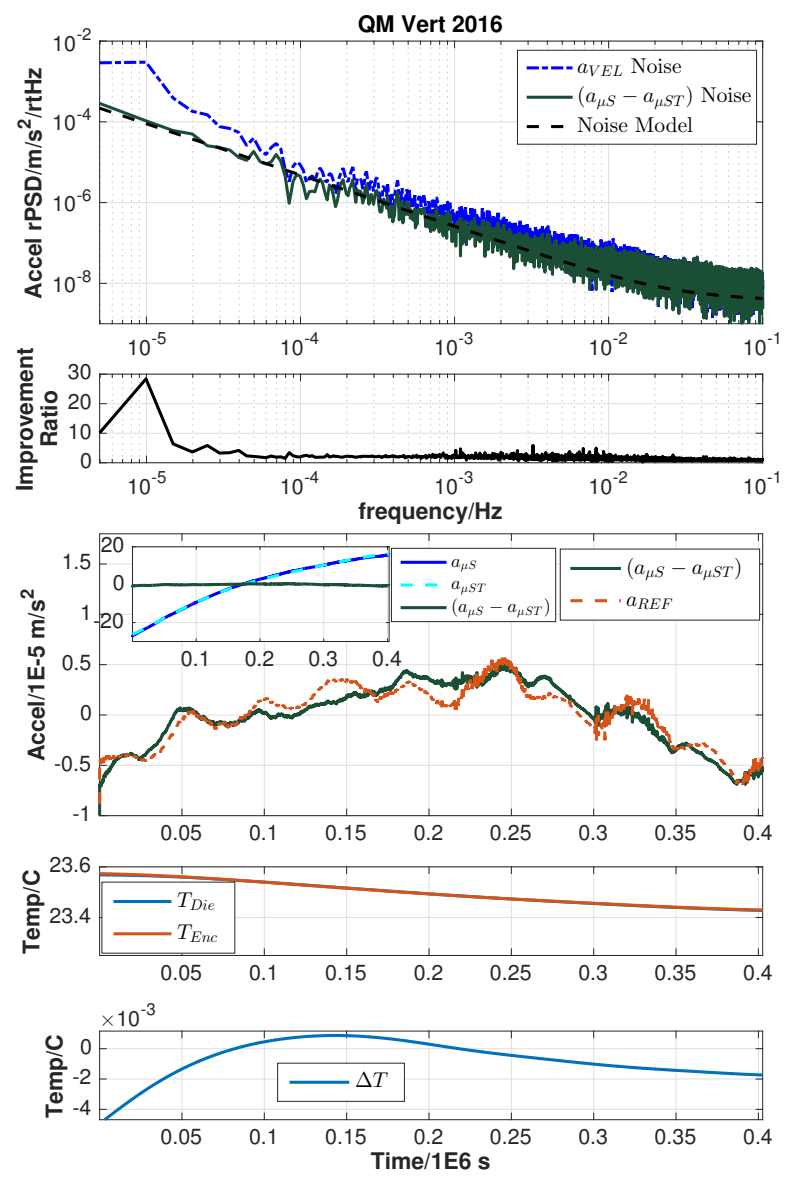

Figure 8: QM Vert 2016. First pane: Self-noise determination from the $a_{V E L}$ and temperature decorrelated, $a_{\mu S}-a_{\mu S T}$, signals. Second pane: Improvement ratio between self-noise before and after temperature decorrelation. Third Pane: acceleration time series for the reference, $a_{R E F}$, and decorrelated microseismometer acceleration with a comparison of the $\mathrm{K}-\mathrm{TF}+\mathrm{T}$ synthesised acceleration and thermal model fit inset. Fourth pane: $T_{d i e}$ and $T_{e n c}$ signals. Fifth pane: $\Delta T$ signal.

applied to a MEMS microseismometer designed for the NASA InSight mission to Mars. The novel approach utilises the complementary properties of a seismometer's mass position and velocity outputs to counteract the ill-posedness of current conventional methods. Furthermore, a thermal model of the microseismometer's acceleration has been introduced. It has been shown that this can be integrated into the transferfunction correction method and implemented for thermal decorrelation of the microseismomter's acceleration signal. The proposed methods are based on obtaining an accurate full-band acceleration response in order to remove the thermal contribution with a physics-based model. As a result, the method is limited by two aspects: the fidelity/SNR of the acquired data and the range for which the coupling applies for the physics-based model. For example, as shown in Figure 7 , the quantisation of the FM mass position can cause an erroneous fusion for certain bandwidths.

Experimental results confirmed that this technique successfully removes the thermal component from the data for bandwidths $<1 \times 10^{-2} \mathrm{~Hz}$. For the temperature sensitivity tests and the cold test the acceleration signal has been recovered
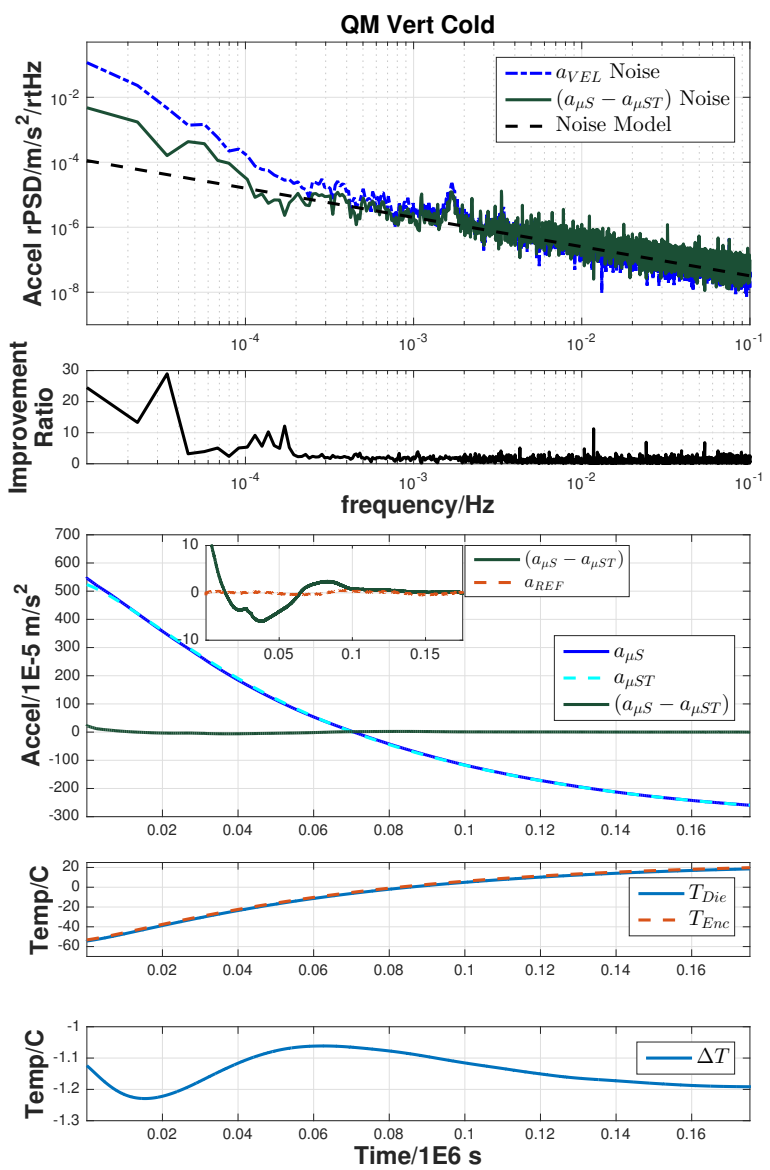

Figure 9: QM Vert Cold. First pane: Self-noise determination from the $a_{V E L}$ and temperature decorrelated, $a_{\mu S}-a_{\mu S T}$, signals. Second pane: Improvement ratio between self-noise before and after temperature decorrelation. Third Pane: acceleration time series for the $\mathrm{K}-\mathrm{TF}+\mathrm{T}$ synthesised acceleration, thermal model fit and temperature decorrelated singals with the reference, $a_{R E F}$, and the decorrelated microseismometer acceleration inset. Fourth pane: $T_{d i e}$ and $T_{e n c}$ signals. Fifth pane: $\Delta T$ signal.

to the level of the sensor noise/second-order effects with a thermal attenuation of 25-50. The ambient temperature tests with a reference show that the signal can be recovered to reveal tidal signals with the estimated sensor self-noise following a single power law. The performance of the passive thermal compensation technology incorporated in the sensor is also validated. Such performance characteristics and mass of the microseismometer have been suggested as the baseline for the seismic instrument in NASA's Europa Lander Study by its Science Definition Team [28] [29] [30]. In this and other applications, the demonstrated ability to implement a virtual vault will contribute to the maximum retrieval of seismic information from the sensors.

\section{ACKNOWLEDGEMENTS}

We would like to thank the UK Space Agency for funding.

\section{APPENDIX A}

IMPLEMENTATION OF THE K-TF AND K-TF+T METHODS

In this section we provide MATLAB code and implementation notes for the proposed K-TF and K-TF+T methods introduced in Section IV. Each method implements the Kalman 
filter in Algorithm 1 which requires: (i) a row vector of $N$ samples for the measurements, $\mathbf{z}$, and the forward model inputs $\mathbf{u}$, (ii) the forward model and measurement equation matrices $\mathbf{F}, \mathbf{B}$ and $\mathbf{H}$ and (iii) the noise covariance matrices $\mathbf{Q}$ and $\mathbf{R}$.

The input to the forward model, $\mathbf{u} \in \mathbb{R}^{1 \times N}$, is given by the acceleration response derived from the velocity channel, $a_{V E L}$, for both the K-TF and K-TF+T methods. To obtain the $N$ data samples, the logged data are first scaled by a gain factor and the transfer-function is provisionally corrected using the frequency division method described in Section III-B which yields the velocity response to be differentiated. For the KTF method the measurement samples $\mathbf{z} \in \mathbb{R}^{1 \times N}$ are given by $a_{P O S}$, which is obtained from a scaling of the raw mass position outputs by a gain factor. The frequency response does not need to be corrected as it is flat in the required bandwidth. The K-TF+T method also requires the calibrated temperature signals $T_{d i e}$ and $\Delta T$ which are concatenated to give the measurement samples data matrix $\mathbf{z}=\left[T_{\text {die }}, \Delta T, a_{P O S}\right]^{\top} \in$ $\mathbb{R}^{3 \times N}$

In the $\mathrm{K}$-TF method, the matrices $\mathbf{F} \in \mathbb{R}^{2 \times 2}, \mathbf{B} \in \mathbb{R}^{2 \times 1}$, and $\mathbf{H} \in \mathbb{R}^{1 \times 2}$ are given in (9) and (11) whereas for the $K$ $\mathrm{TF}+\mathrm{T}$ method, the forward model $(\mathbf{F}$ and $\mathbf{B})$ is unchanged but the measurement model matrix $\mathbf{H} \in \mathbb{R}^{3 \times 2}$ is given in (13). The noise covariance matrices $\mathbf{Q}=\sigma_{F M}^{2} \mathbf{I}$ and $\mathbf{R}=\sigma_{M}^{2} \mathbf{I}$ are modelled as identity matrices scaled by the noise variances, $\sigma_{F M}^{2}$ and $\sigma_{M}^{2}$ which are determined depending on the desired mixture of the two predictions.

Listing 1 provides code to provide the inputs for the Kalman filter for the K-TF method. Listing 2 shows the updated measurement equation required for the Kalman filter inputs in the $\mathrm{K}-\mathrm{TF}+\mathrm{T}$ method. The output state vector, $\mathrm{x}$, consists of the estimate of the microseismometer acceleration response, $a_{\mu S}$, and the correction error, $a_{e r r}$. In Listing 2 , the code is given for the least-squares regression to recalculate the thermal model parameters from the $\mathrm{K}-\mathrm{TF}+\mathrm{T}$ output. This code can be used to implement Algorithm 2.

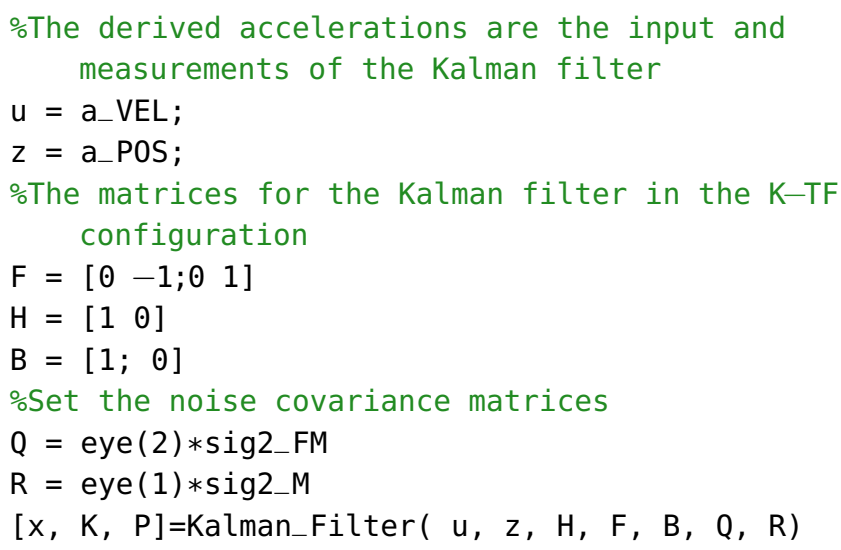

Listing 1: Code to implement the K-TF method for transfer-function inversion.

\section{REFERENCES}

[1] W. Banerdt, S. Smrekar, P. Lognonné, T. Spohn, S. Asmar, D. Banfield, L. Boschi, U. Christensen, V. Dehant, W. Folkner et al., "InSight: a discovery mission to explore the interior of Mars," in Lunar and Planetary Science Conference, vol. 44, 2013, p. 1915.

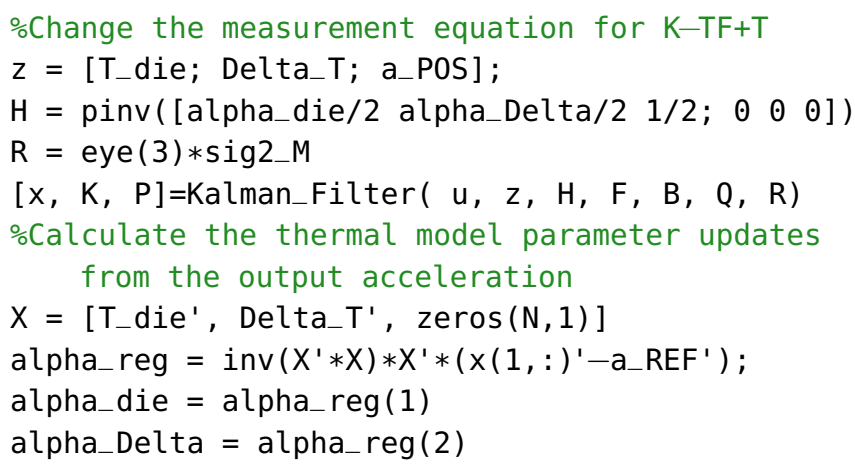

Listing 2: Code to update the measurement equation for the K-TF+T method and calculate the thermal model coefficients.

[2] W. Pike, A. Delahunty, A. Mukherjee, G. Dou, H. Liu, S. Calcutt, and I. Standley, "A self-levelling nano-g silicon seismometer," in SENSORS, 2014 IEEE. IEEE, 2014, pp. 1599-1602.

[3] W. Pike, S. Calcutt, I. Standley, A. Mukherjee, J. Temple, T. Warren, C. Charalambous, H. Liu, A. Stott, and J. McClean, "A Silicon Seismic Package (SSP) for Planetary Geophysics," in Lunar and Planetary Science Conference, vol. 47, 2016, p. 2081.

[4] W. T. Pike, S. M. Calcutt, I. M. Standley, and A. G. Mukherjee, "A Broad-band Silicon Microseismometer with $0.25 \mathrm{ng} / \mathrm{rthz}$ performance," in MEMS, 2018 IEEE. IEEE, 2018.

[5] R. Middlemiss, A. Samarelli, D. Paul, J. Hough, S. Rowan, and G. Hammond, "Measurement of the Earth tides with a MEMS gravimeter," Nature, vol. 531, no. 7596, p. 614, 2016.

[6] R. P. Middlemiss, S. G. Bramsiepe, R. Douglas, S. Hild, J. Hough, D. J. Paul, A. Samarelli, S. Rowan, and G. D. Hammond, "Microelectromechanical system gravimeters as a new tool for gravity imaging," Phil. Trans. R. Soc. A, vol. 376, no. 2120, p. 20170291, 2018.

[7] D. Mimoun, N. Murdoch, P. Lognonné, K. Hurst, W. T. Pike, J. Hurley, T. Nébut, W. B. Banerdt, S. Team et al., "The Noise Model of the SEIS Seismometer of the InSight Mission to Mars," Space Science Reviews, vol. 211, no. 1-4, pp. 383-428, 2017.

[8] N. Murdoch, D. Mimoun, R. F. Garcia, W. Rapin, T. Kawamura, P. Lognonné, D. Banfield, and W. B. Banerdt, "Evaluating the windinduced mechanical noise on the InSight seismometers," Space Science Reviews, pp. 1-27, 2016.

[9] N. Murdoch, B. Kenda, T. Kawamura, A. Spiga, P. Lognonné, D. Mimoun, and W. B. Banerdt, "Estimations of the seismic pressure noise on Mars determined from Large Eddy Simulations and demonstration of pressure decorrelation techniques for the InSight mission," Space Science Reviews, pp. 1-27, 2017.

[10] H. Liu and W. Pike, "A silicon/solder bilayer thermal actuator for compensating thermal drift of silicon suspensions," in Solid-State Sensors, Actuators and Microsystems (TRANSDUCERS), 2015 Transducers-2015 18th International Conference on. IEEE, 2015, pp. 916-919.

[11] P. Goldstein and A. Snoke, "Sac availability for the iris community," Incorporated Research Institutions for Seismology Newsletter, vol. 7, no. UCRL-JRNL-211140, 2005.

[12] M. Freybourger, J. Hinderer, and J. Trampert, "Comparative study of superconducting gravimeters and broadband seismometers STS-1/Z in seismic and subseismic frequency bands," Physics of the earth and planetary interiors, vol. 101, no. 3-4, pp. 203-217, 1997.

[13] S. M. Riad, "The deconvolution problem: An overview," Proceedings of the IEEE, vol. 74, no. 1, pp. 82-85, 1986.

[14] G. Helffrich, J. Wookey, and I. Bastow, The Seismic Analysis Code: A Primer and User's Guide. Cambridge University Press, 2013.

[15] C. D. Rodgers, Inverse methods for atmospheric sounding: theory and practice. World scientific, 2000, vol. 2 .

[16] A. Assa and F. Janabi-Sharifi, "A kalman filter-based framework for enhanced sensor fusion," IEEE Sensors Journal, vol. 15, no. 6, pp. 3281$3292,2015$.

[17] R. C. Luo, C. C. Chang, and C. C. Lai, "Multisensor fusion and integration: Theories, applications, and its perspectives," IEEE Sensors Journal, vol. 11, no. 12, pp. 3122-3138, 2011.

[18] M. P. Panning, S. C. Stähler, H.-H. Huang, S. D. Vance, S. Kedar, V. C. Tsai, W. T. Pike, and R. D. Lorenz, "Expected seismicity and the seismic noise environment of europa," Journal of Geophysical Research: Planets, vol. 123, no. 1, pp. 163-179, 2018. 
[19] E. Wielandt and G. Streckeisen, "The leaf-spring seismometer: Design and performance," Bulletin of the Seismological Society of America, vol. 72, no. 6A, pp. 2349-2367, 1982.

[20] R. Sleeman, A. Van Wettum, and J. Trampert, "Three-channel correlation analysis: A new technique to measure instrumental noise of digitizers and seismic sensors," Bulletin of the Seismological Society of America, vol. 96 , no. 1, pp. 258-271, 2006.

[21] L. G. Holcomb, "A direct method for calculating instrument noise levels in side-by-side seismometer evaluations," HISTORY, vol. 2, p. 1, 1989.

[22] A. Ringler and C. Hutt, "Self-noise models of seismic instruments," Seismological Research Letters, vol. 81, no. 6, pp. 972-983, 2010.

[23] A. Barzilai, T. VanZandt, and T. Kenny, "Technique for measurement of the noise of a sensor in the presence of large background signals," Review of scientific instruments, vol. 69, no. 7, pp. 2767-2772, 1998.

[24] D. P. Mandic, S. Kanna, and A. G. Constantinides, "On the intrinsic relationship between the least mean square and kalman filters [lecture notes]," IEEE Signal Processing Magazine, vol. 32, no. 6, pp. 117-122, 2015.

[25] Y. S. Suh, "Orientation estimation using a quaternion-based indirect kalman filter with adaptive estimation of external acceleration," IEEE Transactions on Instrumentation and Measurement, vol. 59, no. 12, pp. 3296-3305, 2010.

[26] H. Zhao and Z. Wang, "Motion measurement using inertial sensors, ultrasonic sensors, and magnetometers with extended kalman filter for data fusion," IEEE Sensors Journal, vol. 12, no. 5, pp. 943-953, 2012.

[27] T. Warren, W. Pike, S. Calcutt, N. Bowles, and J. Temple, "Cold noise testing of a microseismometer for planetary and terrestrial applications," in AGU Fall Meeting Abstracts, 2016.

[28] K. Hand, A. Murray, J. Garvin, W. Brinckerhoff, B. Christner, B. Edgett, B. Ehlmann, C. German, A. Hayes, T. Hoehler, S. Horst, J. Lunine, K. Nealson, C. Paranicas, B. Schmidt, D. Smith, A. Rhoden, M. Russell, A. Templeton, P. Willis, R. Yingst, C. Phillips, M. Cable, K. Craft, A. Hofmann, T. Nordheim, R. Pappalardo, and The Project Engineering Team, "Report of the europa lander science definition team," 2017.

[29] W. Pike, I. Standley, S. Calcutt, S. Kedar, S. Vance, and B. Bills, "The europa seismic package (esp): 1. selecting a broadband microseismometer for ocean worlds." in 3rd International Workshop on Instrumentation for Planetary Mission, vol. 1980, 2016.

[30] S. Vance, S. Kedar, M. Panning, S. Stähler, B. Bills, R. Lorenz, H.H. Huang, W. T. Pike, J. C. Castillo, P. Lognonné, V. Tsai, and A. R. Rhoden, "Vital Signs: Seismology of ocean worlds," Astrobiology, vol. 18, no. 1, pp. 37-53, 2018. 